\title{
Conference Summary and Recent Advances: the 8th Conference on Metal Toxicity and Carcinogenesis
}

\author{
Xixi Zhou $^{1} \cdot$ Scott W. Burchiel ${ }^{1} \cdot$ Laurie G. Hudson $^{1} \cdot \operatorname{Ke~Jian~Liu~}{ }^{1}$
}

Received: 6 May 2015 / Accepted: 7 May 2015 /Published online: 16 May 2015

(C) Springer Science+Business Media New York 2015

\section{Introduction}

Diseases caused by occupational and environmental exposure to metals are a significant public health concern [1-5]. This series of metal toxicity and carcinogenesis conferences has been organized biennially since 2000 $[6,7]$. It has been an important and unique meeting for scientists in the field to exchange and disseminate information regarding metal-induced toxicity and carcinogenicity. The 8th Conference on Metal Toxicity and Carcinogenesis was held in Albuquerque, NM, USA, on October 26-29, 2014, with approximately 130 participants. There were six scientific sessions covering major areas of research in the field: (1) human and population studies, (2) metal carcinogenesis, (3) molecular and cellular mechanisms, (4) metals and mixtures, (5) metabolism and biological targets, and (6) developmental and epigenetic effects. In addition, there was a poster session and an NIH grant workshop given by Danielle J. Carlin from National Institute of Environmental Health Sciences (NIEHS). Here, we summarize the research presented at the conference and highlight the latest advances in the field published in this special issue of Biological Trace Element Research.

Ke Jian Liu

KLiu@salud.unm.edu

1 Department of Pharmaceutical Sciences, College of Pharmacy, University of New Mexico Health Sciences Center, Albuquerque, NM 87131, USA

\section{Summary of Sessions}

\section{Human and Population Studies}

The first session on human and population studies had eight lectures which covered various environmental metal elements and metal-related human health issues. Johnnye Lewis (University of New Mexico, NM, USA) opened this session with the keynote presentation about recent findings in Navajo birth cohort study (NBCS). Her research group worked with Navajo community in testing uranium distribution in water sources in the Navajo area, since uranium was extensively mined in this region in past decades. Analysis of blood and urine samples of parents and infants enrolled in the NBCS showed that uranium is detectable in the urine of newborn babies, which represents a concern since uranium is not acknowledged to cross the placental barrier. Other metals such as arsenic, lead, and mercury were also detectable in newborns. Furthermore, Dr. Lewis is following up these preliminary findings with an ongoing study to link the data of exposure, biomonitoring, and health outcome. Anu Mudipalli (US Environmental Protection Agency, USEPA) then talked about human health risk assessments particularly relevant to early life metal exposures associated with later life disease risk. She not only provided available evidence but also raised questions regarding the need for more studies to understand the relationship of early life exposure (pregnancy/fetal) and later life outcomes (immediate postnatal and adulthood) in order to inform regulatory decisions. Faruque Parvez (Columbia University, NY, USA) reported the work of a population-based Health Effects of Arsenic Longitudinal Study (HEALS) in Bangladesh. They measured arsenic exposure by analyzing arsenic concentration in well water and urine samples. Furthermore, they measured respiratory symptoms, lung function, and mortality of lung 
diseases. These analyses showed that arsenic exposure positively associates with respiratory symptoms. The extensive statistical analysis on disease and mortality data revealed that arsenic exposure in water at low to moderate dose increased the risk of various respiratory outcomes and mortality. Jiangang Shen from The University of Hong Kong reviewed toxic metal element contamination in herbal medicine and therapeutic components of herbal productions. He presented evidence that contamination of metal elements including arsenic, mercury, and lead come not only from the exposure during herbal growth but also from the process of preparing herbal products. Although metal materials such as arsenic trioxide are accepted for certain therapeutic components, the safety of toxic metal elements in herbal medicine and therapeutic components of herbal products needs more attention. Scott Burchiel (University of New Mexico, NM, USA) presented recent findings of his research group about immunosuppression of trivalent inorganic arsenic (arsenite) and monomethylated arsenic (MMA) in humans. He reported that in a small percentage of donors, the proliferation of human peripheral blood mononuclear cells was strongly inhibited by environmental levels of arsenite (1-100 nM). The immunosuppressive effect of MMA is seen in virtually all donors. The preliminary data from blood donors in the Columbia University Bangladesh HEALS Cohort showed that inhibition of T cell proliferation is associated with drinking water and diet exposure as well as urinary arsenite and MMA. These results suggested that the human immune system may be very sensitive to arsenic exposure in drinking water and diet. Paul B. Tchounwou (Jackson State University, MS, USA) then gave a presentation talking about molecular mechanisms of arsenic trioxide (ATO) in the chemotherapy of acute promyelocytic leukemia. He reported that oxidative stress, upregulation of p53 signaling, and DNA damage are molecular mechanisms of ATO cytotoxicity which lead to apoptotic death in cancer cells during ATO pharmacotherapy. Mohammad Rahbar (University of Texas, TX, USA) reported on studies analyzing environmental factors associated with blood lead concentrations in Jamaican children. This population study reported that the geometric mean blood lead concentration in Jamaican children has dropped by $62 \%$ during the past two decades but still remains about two times higher than children in the USA. This work also identified several significant risk factors for higher blood lead concentrations such as residence near high-traffic roads, lower parental education levels, and consumption of the fruit ackee. The awareness of these factors was suggested to help further decrease the level of lead exposure in Jamaican children. Malek El Muayed (Northwestern University, IL, USA) concluded the human and population studies session by reporting on the results about levels of various transition metals in human insulin-producing islets of Langerhans from the general US population. There is a reported association between metal exposure and type 2 diabetes. The results showed that for the average islet total metal content, nickel was the highest at about $235 \mathrm{nmol} / \mathrm{g}$ protein, cadmium was lower at about $30 \mathrm{nmol} / \mathrm{g}$ protein, and arsenic and lead were low at 5.6 and $4.5 \mathrm{nmol} / \mathrm{g}$ protein, respectively. In addition, it was reported that cadmium content in islets from women was significantly higher than those from men.

\section{Metal Carcinogenesis}

The metal carcinogenesis session included a keynote lecture and regular and short presentations covering carcinogenesis of arsenic, chromium, cadmium, and other metals through various mechanisms including regulation of reactive oxygen species (ROS) generation, protein binding, and epigenetic alteration. Xianglin Shi (University of Kentucky, KY, USA) started this session with his keynote lecture about oxidative stress in hexavalent chromium $\mathrm{Cr}(\mathrm{VI})$ carcinogenesis. He talked about an interesting mechanism of differential ROS generation by $\mathrm{Cr}(\mathrm{VI})$ in normal and transformed BEAS-2B cells. In non-transformed cells, $\mathrm{Cr}(\mathrm{VI})$ generated ROS via activation of NADPH oxidase (NOX), which induced apoptosis. But in $\mathrm{Cr}(\mathrm{VI})$-transformed cells, there is an integrated mechanism of upregulation of p62 and Nrf2, decreased ROS generation, apoptosis resistance, and increased cell invasion and migration. Guided by this dual-effect mechanism, various natural compounds were screened. He reported that luteolin, a natural compound, has properties to attenuate $\mathrm{Cr}(\mathrm{VI})$-induced carcinogenesis. Christy Bridges (Mercer University, GA, USA) then reported results suggesting that the breast cancer resistance protein (Bcrp), in addition to multidrug resistance protein 2 (Mrp2), may play a role in the export of mercury via proximal tubular cells of the kidney. This may serve as an important molecular mechanism of inorganic mercury toxicity in the kidney. Chengfeng Yang (Michigan State University, MI, USA) discussed a microRNA-related mechanism in arsenic carcinogenesis. MicroRNA-200b, a member of miR-200 family, targets protein kinase $\mathrm{C} \alpha$ and Wnt5b-PKC $\alpha$ positive feedback loop and subsequently inhibits Rac1 activation, thereby suppressing arsenic-transformed cell migration. Bernard Futscher (University of Arizona, AZ, USA) then presented the results revealing an epigenetic-dysfunction mechanism of arsenic carcinogenesis. In arsenic-transformed cells, DNA hypermethylation-linked $\mathrm{H} 3 \mathrm{~K} 9 \mathrm{me} 3$ widely silenced $\mathrm{C} 2 \mathrm{H} 2$ zinc finger genes (ZNFs). The 10-min short presentations covered various mechanisms of metal carcinogenesis and included sustained over-expression of IL-6 inhibiting autophagy in arsenic carcinogenesis (Gang Chen, University of Kentucky, KY, USA), microRNA-mediated heavy metal toxicity (Yong $\mathrm{Li}$, University of Louisville, KY, USA), apoptosis resistance by Nrf2/p62 signaling in cadmium carcinogenesis (Young-Ok Son, University of Kentucky, KY, USA), dual role of cadmium on LC3B and autophagy in non-transformed and transformed cells in cadmium-induced prostate carcinogenesis 
(Chendil Damodaran, University of Louisville, KY, USA), accumulation of cancer stem-like cells in chronic nickelinduced malignant transformation cells (Lei Wang, University of Kentucky, KY, USA), arsenic binding to different cysteine sites of Keap1 to regulate Nrf2/Keap1 signaling (Xiaoqing He, West Virginia University, WV, USA), ROS production induced by doped cerium oxide nanoparticles (Katherine Dunnick, West Virginia University, WV, USA), and arsenic modulates distinct changes in chromatin structure, gene expression, and splicing through inhibition of PARP-1 DNA binding (Yvonne Fondufe-Mittendorf, University of Kentucky, KY, USA).

\section{Molecular and Cellular Mechanisms}

The second day of the conference started with a molecular and cellular mechanism session including 11 presentations. John Wise (University of Southern Maine, ME, USA) opened this session by reporting his research work on the impact of $\mathrm{Cr}(\mathrm{VI})$ on wild great whales. $\mathrm{Cr}(\mathrm{VI})$ skin levels are dramatically elevated in even the most remote ocean locations. The follow-up cellular study demonstrated that $\mathrm{Cr}(\mathrm{VI})$ is toxic to whale cells. Both particulate and soluble forms of $\mathrm{Cr}(\mathrm{VI})$ induced concentration-dependent increases in cytotoxicity and genotoxicity. The wildlife data from his group suggested that $\mathrm{Cr}(\mathrm{VI})$ has become a global pollutant spread by wind, water, and biological currents. Fei Chen (Wayne State University, MI, USA) reported his molecular mechanism study of arsenic-induced malignant transformation of the lung cells. Arsenic activated JNK, STAT3, and Akt signaling and thus induced mineral dust-induced gene (mdig) expression, which is a newly acknowledged oncogene in lung cancer. The function of mdig was further confirmed utilizing heterozygotic mdig gene knockout mice. Genetic deficiency of mdig weakened Th17 cell infiltration but enhanced Treg cell infiltration and thus altered the balance of Th17 and Treg cells. The results suggested that mdig is a key regulator of Th17 cell function and lung fibrosis in a kinase-dependent manner. Michael Aschner (Albert Einstein College of Medicine, NY, USA) reported the role of dopamine in manganese-induced neurodegeneration. This study performed in C. elegans demonstrated that extracellular dopamine (DA) is responsible for Mninduced dopaminergic (DAergic) neurodegeneration. These results suggest that in vivo BLI-3 activity promotes the conversion of extracellular DA into toxic reactive species, which, in turn, can be taken up by DAT-1 in DAergic neurons, thus leading to oxidative stress and neurodegeneration. The molecular mechanism was reported. Extracellular DA is converted into toxic reactive species promoted by NADPH dual-oxidase BLI-3. Thus, DA-reuptake transporter (DAT-1) takes up those reactive species in DAergic neurons, leading to oxidative stress and neurodegeneration. Keshav Singh (University of Alabama, AL, USA) gave a presentation on molecular genetic analysis of arsenic-induced malignant transformation of epithelial cells. Utilizing an in vitro model of prostate tumorigenesis and comparative genomic hybridization arrays, they found that chromosomes $1,7,9,12$, and 18-20 have deletions and chromosomes $8,9,11,18$, and 20 have regional amplifications. They intend to follow up these analyses with dissecting novel oncogenes and tumor suppressor genes in prostate tumorigenesis by arsenic. Christopher States (University of Louisville, KY, USA) presented a molecular mechanism regarding how cancers overcome arsenic disruption of mitotic progression. They have shown that $\mathrm{p} 53-\mathrm{CDKN} 1 \mathrm{~A}$ pathway contributes to the escape of mitotic blockade which is associated with inadequate degradation of securin. From arsenicexposed patients, analyses of $\mathrm{p} 53$ genes suggested that $\mathrm{p} 53$ was not mutated, but microRNAs that target securin were induced. These findings suggested that arsenic-induced cancers overcome the mitosis-disrupting effects of arsenic through maintenance of $\mathrm{p} 53$ function and induction of microRNAs. Dongyun Zhang (New York University, NY, USA) reported on a molecular mechanism in arseniteinduced cell death. During cellular response to oxidative stress, nucleolin, a cell cycle regulator, maintains its nuclear localization and messenger RNA (mRNA) binding activity through SUMOylation at K294, a novel posttranslational modification of nucleolin. Under oxidative stress conditions induced by arsenic exposure, nucleoli SUMOylation promoted nucleolin binding with gadd45a mRNA; increased gadd45a mRNA stabilization and protein expression led to cell death. On the other hand, gadd45a expression was demonstrated to be reduced through decreased superoxide level by MnSOD and inhibition of nucleolin SUMOylation, which contributed to the inhibition of arsenic-induced apoptosis. These findings suggested that the novel posttranslational modification of nucleolin is regulated by both redox and proproliferative signals and has dual effects in cancer development. Andrea Allan (University of New Mexico, NM, USA) presented her work on the molecular mechanisms of arsenic effects on mood, learning, and memory disorders in mice, in which the glucocorticoid system seems to play a critical role. This study for the first time suggested that prenatal arsenic exposure causes prolonged developmental imbalance between two $11 \beta$-hydroxysteroid dehydrogenase enzymes, 11ßHSD1 and $11 \beta \mathrm{HSD} 2$, thus leading to alteration of glucocorticoid receptor (GR) expression. Furthermore, GR signaling alteration throughout adulthood was suggested to be the consequence of gestational arsenic exposure-induced alteration of GR and 11ßHSD2 profiles. Suthakar Ganapathy (Northeastern University, MA, USA) presented a novel mechanism for protection of normal cells in chemo/radiation therapy by arsenic. Low-dose arsenic reduces chemotherapy-induced toxicity in normal cells via induction of glycolysis, which is mediated by $\mathrm{p} 53 / \mathrm{NFKB} / \mathrm{HIF}-1 \alpha$ pathway. The significance of this research was that normal functional p53 is required for the 
protective effect of arsenic; therefore, normal cells could be protected rather than cancer cells.

Lori Peterson (Caldera Pharmaceuticals, Inc.) introduced $\mathrm{XRpro}^{\circledR}$ molecular X-ray fluorescence $\left(\mathrm{MXRF}^{\circledR}\right)$ to the audience. It is a high-throughput method used for screening metalselective ligands. Application examples were presented such as selectively quantifying $\mathrm{Cr}(\mathrm{VI})$ vs $\mathrm{Cr}(\mathrm{III})$ ligands and analysis of gadolinium (Gd) ligands which do not release this metal under in vivo conditions. Astrid Engel (Tulane University, LA, USA) presented their studies on the differential influence on double-strand break (DSB) DNA repair outcomes by nickel, cadmium, and arsenic exposures. Utilizing two complementary ex vivo assay systems, the choice of DSB DNA repair pathways and the repair outcomes under heavy metal exposure was quantitatively determined. Under nickel and cadmium exposure, resolution of DSBs was mostly through homologous recombination. In contrast, under arsenic exposure, cells preferentially repaired DSBs through nonhomologous end joining. The metal dose also altered selection of DSB repair pathways. This work demonstrated that cells repair DSBs in a dose-dependent manner that is specific to a particular metal. The last presentation in this session was given by Sandra Wise (University of Southern Maine, ME, USA), answering the question of whether particulate $\mathrm{Cr}(\mathrm{VI})$ induced chromosome instability (CIN) is a transient or a permanent phenotype change. She performed a series of experiments including human lung cells exposed to lead chromate then seeded cells after each treatment at colony-forming density, cloned, expanded, and retreated. Each generation of clones was tested for chromosome complement, DSB repair efficiency, centrosome amplification, and its ability to grow in soft agar. Repair-deficient clones increasingly showed up in this analysis. Further investigation revealed that repairdeficient clones were unable to form Rad51 foci in response to radiation, indicating a defect in homologous recombination repair. In addition, many of the clones exhibited centrosome amplification. Many clones formed colonies in soft agar suggesting that the cells had neoplastically transformed. This work concluded that particulate $\mathrm{Cr}(\mathrm{VI})$ induces a permanent phenotypic change of CIN and DSB repair deficiency in human lung cells.

\section{Metals and Mixtures}

The scientific session in the second afternoon of the conference focused on metals and mixtures. Six presentations talked about health and disease outcomes of various metals such as arsenic, cadmium, zinc, uranium, and mixtures. Laurie Hudson (University of New Mexico, NM, USA) opened this session with the keynote presentation on the mechanism of arsenic inhibition of DNA repair. Two major hypotheses were acknowledged as possible molecular mechanisms of DNA repair inhibition by arsenic: Arsenic induced ROS generation and disruption of $\mathrm{C} 3 \mathrm{H} 1$ and $\mathrm{C} 4$ zinc finger DNA repair targets by selective binding. Here, she reported that in the presence of arsenic, $\mathrm{C} 3 \mathrm{H} 1$ and $\mathrm{C} 4$ zinc finger proteins were selectively oxidized. The mechanism was that in contrast to zinc, arsenic binding with zinc finger motif fails to protect against oxidation. The findings demonstrated that selective arsenic binding to zinc fingers confers selective oxidation of cysteine residues within the zinc finger protein targets and thus provides a mechanistic basis linking the arsenic binding and oxidative damage selectivity on zinc finger structure. Diane Blake (Tulane University, LA, USA) then gave a presentation about their research on the mixture of cadmium and benzo[a]pyrene $(\mathrm{B}[\mathrm{a}] \mathrm{P})$. In a novel cell line derived from healthy adult human renal proximal tubule epithelial cells (RPTEC) immortalized using human telomerase reverse transcriptase (TERT1) as a model of renal cell carcinomas in kidney cancers, preexposure to a non-cytotoxic concentration of $\mathrm{Cd}$ significantly reduced DNA adducts induced by $\mathrm{B}[\mathrm{a}] \mathrm{P}$ exposure. Furthermore, co-exposure of $\mathrm{Cd}$ and $\mathrm{B}[\mathrm{a}] \mathrm{P}$ elevated Nrf2-responsive genes such as GCLC, HMOX1, and NQO1. Therefore, this work demonstrated Cd's ability to induce antioxidant pathways, which may lead to attenuation of $\mathrm{B}[\mathrm{a}] \mathrm{P}$ DNA adduct formation in RPETC/TERT1 cells. In contrast to environmental toxicants like cadmium and arsenic, zinc is a necessary nutrient. Emily Ho (Oregon State University, OR, USA) discussed the biological consequence of zinc deficiency. She reported that insufficient zinc intake caused oxidative stress and DNA damage and impaired DNA repair mechanisms. Thus, zinc deficiency could increase one's susceptibility to environmental toxins such as arsenic. On the other hand, zinc repletion could reverse DNA damage induction and protect DNA integrity against environmental toxins. While most studies focus on soluble metal compounds, Matthew Campen (University of New Mexico, NM, USA) drew attention to the toxicity of metal components in ambient airborne particulate. Inhalation of particulate matter rich in metals such as vanadium, iron, and nickel is associated with cardiovascular morbidity and mortality in epidemiological and toxicological studies. He reported that vanadium and nickel synergistically drive cardiac arrhythmias and that nickel was capable of enhancing atherosclerosis and vascular dysfunction. The public health concern of insoluble forms of metal exposure was demonstrated and investigated in this work. Diane Stearns (Northern Arizona University, AZ, USA) reported the synergistic toxicity of uranium and UVB radiation. Her results demonstrated that cellular exposure of uranyl acetate (UA), followed by UVB radiation, caused more cytotoxic and mutagenic effects in Chinese hamster ovary cells than individual exposures. The synergistic effect depended on order of treatment. Arsenic and cadmium did not show comparable synergistic cytotoxicity with UVB. These results provided a new model of uranium action suggesting that photo-activated uranyl ion may be more damaging, which also indicated the skin to be a 
target organ of uranium toxicity. Melissa Badding (National Institute for Occupational Safety and Health, NIOSH, WV, USA) talked about indium-tin oxide (ITO)-induced cytotoxicity and inflammatory responses. The results showed that sintered ITO (SITO) not only causes cell death but also activates cytokine production such as IL- $1 \beta$, IL- 6, TNF $\alpha$, and IL8 through NFKB signaling pathway, which suggests inflammasome activation by SITO exposure.

\section{Metabolism and Biological Targets}

The last day of the conference had two sessions, one of which focused on metal metabolism and related biological targets. John B. Vincent (University of Alabama, AL, USA) opened this session by his keynote presentation about $\mathrm{Cr}$ (III) transport. It is critical in toxicology studies and pharmacology applications of $\mathrm{Cr}(\mathrm{III})$ to understand the distribution, elimination, and transport of $\mathrm{Cr}(\mathrm{III})$ in vivo. Transferrin and lowmolecular-weight chromium-binding substance (LMWCr) are known to transport $\mathrm{Cr}$ (III). Kinetic studies on $\mathrm{Cr}$ (III) binding to transferrin did not show physiologically relevant reaction speeds favorable for binding. LMWCr-related results were also presented. The following three presentations focused on arsenic but from diverse perspectives. Walt Klimecki (University of Arizona, AZ, USA) presented their recent work about arsenic carcinogenesis from the angle of energy metabolism disruption. In BEAS-2B cells, non-cytotoxic arsenic exposure induced aerobic glycolysis, increased mitochondria mass, and targeted pyruvate dehydrogenase, thus accumulating intracellular pyruvate and lactate. Furthermore, arsenic exposure decreased E-cadherin expression, a hallmark of malignancy, and accumulated HIF-1 $\alpha$, which contributed to upregulation of HIF $1 \alpha$-target genes. These findings suggested an integrated mechanism of arsenic-induced malignant transformation with the participation of energy metabolism perturbation and hypoxic cellular response induction. Koren Mann (McGill University, QC, Canada) talked about arsenicenhanced atherosclerosis from the angle of arsenic metabolism (methylation). Arsenic exposure correlates with atherosclerosis epidemiologically, and monocytes/macrophages are critical to atherosclerosis development; thus, macrophages were suggested to be a target for arsenic. This work indicated that expression of As3MT, which is associated with arsenite methylation, is induced during monocyte/macrophage polarization. Methylated arsenicals enhanced atherosclerosis similar to arsenite. As3MT/ApoE double knockout mice were insensitive to arsenite-enhanced plaque formation. This work suggested that arsenic methylation may be a mechanism of atherosclerosis enhancement in a macrophage-dependent fashion. Laurent Vernhet (Université de Rennes, France) gave a presentation about their research on mechanisms of arsenic immunotoxicity. The study demonstrated that in human monocyte-derived dendritic cells, inorganic arsenic activates
Nrf2 rapidly, leading to an inhibition of IL-12 expression and a direct inhibition of LPS pathways and immunosuppressive effects.

\section{Developmental and Epigenetic Effects}

The last session covered a recent research work on epigenetic effects of arsenic, uranium, and nickel. Max Costa (New York University, NY, USA) gave a keynote presentation about an epigenetic mechanism of arsenic carcinogenesis. Stem-loopbinding protein (SLBP), which regulates canonical histone mRNA metabolism, was downregulated by arsenic exposure leading to dramatic induction of polyadenylated H3.1 mRNA. The detailed mechanism was hypothesized that arsenic depletion of nuclear SLBP results in inhibition of binding of SLBP to H3.1 transcripts, thus facilitating mRNA transcription and increased mRNA half-life, both of which contribute to elevation of H3.1 protein levels. The epigenetic effect of uranium was presented by Alexandra Miller (Armed Forces Radiobiology Research Institute, MD, USA). She reported a mechanism pointing to leukemogenesis of depleted uranium (DU) based on chromosomal instability in bone marrow cells. In DU-exposed DBA/2 mice, there was a persistent genomic instability in the clonal descendants of hematopoietic stem cells. Also, methylation status of several promoter regions was significantly altered by DU exposure. She also reported results from studies on kidney that DU largely altered gene transcription. The presentation about nickel by Michael Maroney (University of Massachusetts, MA, USA) reported an epigenetic mechanism of nickel carcinogenesis, whereby $\mathrm{Ni}(\mathrm{II})$ substitutes non-heme $\mathrm{Fe}(\mathrm{II})$ active sites in multiple DNA and histone demethylases, thus altering the structure and function of these enzymes and gene expression profile.

\section{NIH Grant Workshop}

During the first day of the conference, there was a special NIH grant workshop, which greatly benefited the participants. Danielle Carlin from National Institute of Environmental Health Sciences (NIEHS) gave a presentation about the NIEHS Superfund Research Program (SRP). The goal of the SRP is to improve the understanding of, and minimize the health effects associated with, exposures to environmental contaminants including metals and metal mixture exposures. The SRP supports research from biomedical to environmental science and engineering, as well as community engagement, research translation, and training. The presentation covered recent findings in metal and metalloid research from the SRP grant portfolio and discussed the links between metals and health outcomes as well as the challenges in the field. Furthermore, potential future directions were discussed, including research on complex metal mixtures, determination of relevant metal exposures, identifying affected 
developmental windows of susceptibility, risk assessment and management, and development of research tools.

\section{In This Special Issue}

This special issue consists of 13 papers, and the findings from most of these papers were presented as an oral presentation and/or poster at the conference. Three papers focus on chromium: John B. Vincent reviews the evidence for $\mathrm{Cr}(\mathrm{III})$ as a second messenger to increase insulin sensitivity and proposes a plan for testing the hypothesis; Catherine F. Wise et al. report $\mathrm{Cr}(\mathrm{VI})$ exposure to fin whale and toxicity to fin whale skin cells, suggesting that $\mathrm{Cr}(\mathrm{VI})$ is a concern for whales in the Gulf of Maine; and Hong Xie et al. compare the cytotoxic and clastogenic effects of $\mathrm{Cr}(\mathrm{VI})$ in human lung and skin fibroblasts, demonstrating that differences in carcinogenic outcome in the lung and skin come from different cellular and molecular responses to $\mathrm{Cr}(\mathrm{VI})$. There are three papers discussing arsenic: J. Christopher States reviews arsenic disruption of mitotic progression as a mechanism of arsenic toxicity and therapeutic applications; Jason Brocato et al. report an epigenetic mechanism of arsenic carcinogenesis based on arsenic upregulated polyadenylated H3.1 mRNA through depletion of stem-loop-binding protein; and Peace C. Ezeh et al. report the interactions between arsenite and dibenzo[def, p]chrysene (DBC) on mouse bone marrow, suggesting a mechanism of arsenic and DBC immunosuppression at environmentally relevant doses. Two papers in this issue discuss nanoparticle toxicity though ROS generation: The work by Katherine M. Dunnick et al. suggests that the transition between the two valence states of cerium and the presence of increased oxygen vacancies, rather than a specific valence state of cerium, play an important role in toxicity of cerium oxide $\left(\mathrm{CeO}_{2}\right)$ nanoparticles; Ling-Zhi Liu et al. report that induction of angiogenic response by activating ROS, AKT, and ERK $1 / 2$ signaling pathways is suggested to be a mechanism of toxicity of tungsten carbide and metallic cobalt (WCCo) nanoparticles. In this special issue, Bridget R. SimonFriedt et al. report an improved tool, the RPTEC/TERT1 cell line, for cadmium toxicity assessments; the mini-review by Maria E. Morales et al. provides some background knowledge on transposable elements (TEs), including their roles in disease, and gave an overview of the current knowledge on how heavy metals influence TE-mediated damage; and V. M. Andrade et al. review the toxicity mechanisms of lead, arsenic, and mercury exposure, as well as mixture exposure, and thus addressed putative biomarkers in risk assessment. There are also two papers related to neuroscience: Lei Zhao et al. report their effort in investigating whether a metal elementcontaining herbal formula, Angong Niuhuang pill, is safe for acute brain disorders; Rong Pan et al. report their work in demonstrating the involvement of autophagy in zinc-induced hypoxic cell death and zinc-potentiated ischemia-reperfusion injury.

\section{Summary}

From the research presented in the 8th Conference on Metal Toxicity and Carcinogenesis, we see emerging hot spots in this field, including the following: the health outcomes of metal mixtures and research of metal mixtures with other contaminants such as PAHs, the epigenetic mechanisms in metal toxicity, the detailed mechanistic study of metal interaction with proteins, and human/population studies linking metal exposure to consequences in human health. However, there are still challenges such as finding and validating biomarkers for metal exposure and related health outcomes and translating the achievements of mechanism-based research into approaches in prevention and intervention of metal toxicity and carcinogenesis. In total, the conference not only covered concepts, approaches, and interventions novel to the field of metal toxicity and carcinogenesis but also stimulated interactions of scientists and collaborations, which should help move the field forward.

Acknowledgments The 8th Conference on Metal Toxicity and Carcinogenesis was supported by a conference grant from the NIH/National Institute for Environmental Health Sciences (R13ES024646) and by the University of New Mexico's College of Pharmacy, Health Sciences Center, Cancer Center (P30CA118100), and Clinical \& Translational Sciences Center (UL1TR000041).

\section{References}

1. Chou S, Harper C, Ingerman L et al (2007) Toxicological profile for arsenic. Agency for Toxic Substances and Disease Registry (US), Atlanta

2. Wilbur S, Abadin H, Fay M et al (2012) Toxicological profile for chromium. Agency for Toxic Substances and Disease Registry (US), Atlanta

3. Faroon O, Ashizawa A, Wright S et al (2012) Toxicological profile for cadmium. Agency for Toxic Substances and Disease Registry (US), Atlanta

4. Koedrith P, Seo YR (2011) Advances in carcinogenic metal toxicity and potential molecular markers. Int J Mol Sci 12:9576-9595. doi: 10.3390/ijms12129576

5. Beyersmann D, Hartwig A (2008) Carcinogenic metal compounds: recent insight into molecular and cellular mechanisms. Arch Toxicol 82:493-512. doi:10.1007/s00204-008-0313-y

6. Bower JJ, Leonard SS, Shi X (2005) Conference overview: molecular mechanisms of metal toxicity and carcinogenesis. Mol Cell Biochem 279(1-2):3-15

7. Leonard SS, Bower JJ, Shi X (2004) Metal-induced toxicity, carcinogenesis, mechanisms and cellular responses. Mol Cell Biochem 255:3-10 